\title{
The Optimal Temperature Control for the Reactions with Parallel Deactivation of Enzyme Encapsulated inside Microorganism Cells
}

\author{
Ireneusz Grubecki \\ Faculty of Chemical Technology and Engineering, University of Technology and Life Sciences \\ 3 Seminaryjna Street, 85-326 Bydgoszcz, Poland \\ e-mail: igrubeck@utp.edu.pl
}

(Received: 30 August 2011; revised: 22 November 2011; accepted: 25 November 2011; published online: 6 December 2011)

\begin{abstract}
A batch biotransformation process running in the presence of microorganisms cells revealing a specified enzyme activity has been considered. A non-linear deactivation model suggested by Do and Weiland has been taken into account for modeling of the process. Based on variational calculus computations aimed at finding an optimal selection of temperature conditions that ensure obtaining maximum conversion or minimum duration time necessary for its attaining have been carried out. The solutions were given and discussed for the stationary process, and for the active upper and lower temperature limitations. It has been proved that an application of microorganisms cells results in slowing down the reaction rate and shifting the initial temperature of the stationary profile to higher values. They are more pronounced the lower the permeability of the cell membrane is. In consequence, an extension of the process duration time is observed along the sections of the optimal profile while the lower temperature constraint usually becomes inactive.
\end{abstract}

Key words: optimization, batch bioreactor, optimal temperature control, biocatalyst deactivation, variational calculus

\section{SYMBOLS}

$C_{i} \quad$ substrate concentration $(i=\mathrm{S})$, substrate concentration inside microorganism cells $(i=\mathrm{SIn})$ and enzyme concentration $(i=\mathrm{E})\left[\mathrm{kmol} \mathrm{m}^{-3}\right]$

$E_{j} \quad$ activation energy for reaction $(j=\mathrm{R})$, deactivation $(j=\mathrm{D})$ and permeability of the cells membrane $(j=\mathrm{P})$ $\left[\mathrm{J} \mathrm{kmol}^{-1}\right.$ ]

E dimensionless quotient of the activation energies $\left(=E_{\mathrm{D}} / E_{\mathrm{R}}\right)$

$k_{j 0} \quad$ frequency factor for reaction $(j=\mathrm{R})$, deactivation $(j=\mathrm{D})$ and permeability of the cells membrane $(j=\mathrm{P})$ $\left[\mathrm{s}^{-1}\right]$

$k_{j} \quad$ constant of reaction rate $(j=\mathrm{R})$, deactivation $(j=\mathrm{D})$ and permeability of the cells membrane $(j=\mathrm{P})\left[\mathrm{s}^{-1}\right]$

$K_{\mathrm{D}} \quad$ Michaelis-Menten constant for deactivation $\left[\mathrm{kmol} \mathrm{m}^{-3}\right]$

$\bar{K}_{\mathrm{D}} \quad$ dimensionless constant for deactivation $\left(=K_{\mathrm{D}} / C_{\mathrm{S} 0}\right)$

$K_{\mathrm{M}} \quad$ Michaelis-Menten constant for reaction $\left[\mathrm{kmol} \mathrm{m}^{-3}\right]$

$\bar{K}_{\mathrm{M}} \quad$ dimensionless constant for reaction $\left(=K_{\mathrm{M}} / C_{\mathrm{S} 0}\right)$

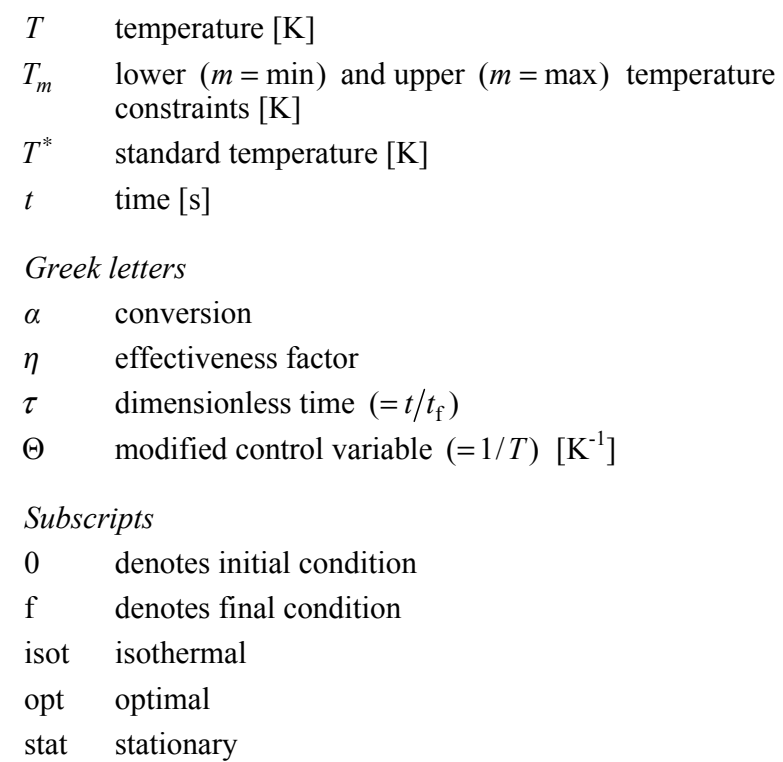




\section{INTRODUCTION}

Batch reactors with deactivating catalysts are widely used for production of various products in chemical and biotechnological industry. In such reactors, high conversion of a substrate can be achieved even at isothermal conditions. However, it can take a long time to accomplish the process and it may lead to the usage of a large amount of biocatalyst. Therefore, in recent years there has been a growing interest in finding a temperature control mode which could enable either the maximization of the economic gain or the minimization of the reaction time [1-3]. At present, the main concern is the selection of temperature conditions for biotransformation processes, because they often replace less economical chemical processes.

The biotransformations can be carried out in the presence of a native enzyme or an enzyme encapsulated in a whole microbial cell. In spite of diminished selectivity, the latter is particularly advantageous since the natural environment increases enzyme stability, and thus decreases enzyme deactivation.

The analysis of the optimal temperatures given in literature has shown that the kinetics of (bio-)catalyst deactivation and mutual relations between the activation energies exert the decisive effect $[4,5]$. The majority of the processes analyzed from the optimal point of view take into consideration the simplest deactivation model, i.e. the first order deactivation independent of a substrate concentration [5-7]. Still the reported models have described the rate of the deactivation occurring under real conditions with accuracy sufficient for industrial practice.

Less often, the optimal conditions have been considered for processes with a catalyst deactivation dependent upon a substrate concentration $[8,9]$.

The determination of the optimal temperature for a biotransformation with an enzyme encapsulated in microbial cells is significantly more difficult than that for a native enzyme. This is because in the former it is necessary to consider a substrate and product transport through a cell membrane.

The effect of internal and external diffusional resistances on the overall rate of the processes carried out in the presence of immobilized enzymes have been reported in literature $[10,11]$. In most cases, the processes have been analyzed for isothermal conditions, either without consideration of a deactivation of a biocatalyst or with assumption of the first order deactivation [12,13]. The models have been solved by using either the combined analytical and numerical techniques [14], or the latter alone [15]. So far, no research has been reported on the optimal temperature control for a biotransformation process in which diffusional phenomena of a deactivating biocatalyst had been considered.

The aim of this work was the determination of the optimal temperatures for a batch process with parallel deactivation of enzyme encapsulated inside microorganisms cells. In development of the model, both the diffusion of the enzyme across a cell membrane and the deactivation of the enzyme were taken into account.

The effect of the diffusional phenomena on the temperature profiles and on the optimal reaction time was also analyzed.

\section{MATHEMATICAL MODEL}

The course of any enzymatic process is dependent on a number of kinetic parameters that characterize both the reaction itself as well as the accompanying deactivation of the applied biocatalyst so that determining the effects of each of them is impossible. When formulating a mathematical model of the analyzed process the following assumptions have been made:

- substrate transport across the cell membrane is an equimolar diffusion process affecting the overall process rate

$$
-\frac{\mathrm{d} C_{\mathrm{S}}}{\mathrm{d} t}=k_{\mathrm{P}}\left(C_{\mathrm{S}}-C_{\mathrm{SIn}}\right)
$$

where $C_{\text {SIn }}$ determines the substrate concentration inside the microorganism cells,

- the rate of reaction $r_{\mathrm{S}}$ occurring inside the microorganism cells is described by the Michaelis-Menten kinetics

$$
r_{\mathrm{S}}=k_{\mathrm{R}} \frac{C_{\mathrm{E}} C_{\mathrm{SIn}}}{K_{\mathrm{M}}+C_{\mathrm{SIn}}}
$$

- an effect of the substrate concentration on the rate of enzyme deactivation $r_{\mathrm{D}}$, in agreement with Do and Weiland theory [16], has been taken into account

$$
r_{\mathrm{D}}=k_{\mathrm{D}} \frac{C_{\mathrm{E}} C_{\mathrm{SIn}}}{\left(K_{\mathrm{D}}+C_{\mathrm{SIn}}\right)}
$$

- the effect of temperature on the reaction rate constant $k_{\mathrm{R}}$ deactivation rate constant $k_{\mathrm{D}}$ and the overall mass transfer coefficient across the cell membrane $k_{\mathrm{P}}$ is expressed by the Arrhenius equation

$$
k_{i}=k_{i 0} \exp \left(-\frac{E_{i}}{R} \Theta\right) \quad(i=\mathrm{D}, \mathrm{P}, \mathrm{R})
$$


where $\Theta=1 / T$ while $E_{\mathrm{D}}, E_{\mathrm{P}}$ and $E_{\mathrm{R}}$ denote the activation energy for deactivation, permeability of the cells membrane and for reaction, respectively,

- the Michaelis-Menten constant for reaction $K_{\mathrm{M}}$ and deactivation $K_{\mathrm{D}}$ are assumed to be independent of temperature because its variation with this parameter can be neglected,

- in spite of the decrease in enzyme activity the rate of the substrate transport across the cell membrane is equal to the reaction rate inside the cells (quasi-steady state assumption).

In the situation, after introducing dimensionless variables

$$
\bar{C}_{i}=C_{i} / C_{i 0}(i=\mathrm{E}, \mathrm{S})
$$

and

$$
\bar{K}_{l}=K_{l} / C_{\mathrm{S} 0} \quad(l=\mathrm{D}, \mathrm{M})
$$

the mathematical model will take the following form

$$
\begin{aligned}
& -\frac{\mathrm{d} \bar{C}_{\mathrm{S}}}{\mathrm{d} t}=k_{\mathrm{R}}^{\prime} \frac{\bar{C}_{\mathrm{E}} \bar{C}_{\mathrm{SIn}}}{\left(\bar{K}_{\mathrm{M}}+\bar{C}_{\mathrm{SIn}}\right)}=f\left[\bar{C}_{\operatorname{SIn}}\left(\bar{C}_{\mathrm{E}}, \bar{C}_{\mathrm{S}}, \Theta\right), \bar{C}_{\mathrm{E}}, \Theta\right] \\
& -\frac{\mathrm{d} \bar{C}_{\mathrm{E}}}{\mathrm{d} t}=k_{\mathrm{D}} \frac{\bar{C}_{\mathrm{E}} \bar{C}_{\mathrm{SIn}}}{\left(\bar{K}_{\mathrm{D}}+\bar{C}_{\mathrm{SIn}}\right)}=g\left[\bar{C}_{\operatorname{SIn}}\left(\bar{C}_{\mathrm{E}}, \bar{C}_{\mathrm{S}}, \Theta\right), \bar{C}_{\mathrm{E}}, \Theta\right]
\end{aligned}
$$

where $k_{\mathrm{R}}^{\prime}=k_{\mathrm{R}} C_{\mathrm{E} 0} / C_{\mathrm{S} 0}$ denotes the modified constant of the reaction rate and

$$
\begin{gathered}
\bar{C}_{\mathrm{SIn}}\left(\bar{C}_{\mathrm{S}}, \bar{C}_{\mathrm{E}}, \Theta\right)=\frac{1}{2}\left[\bar{C}_{\mathrm{S}}-X_{\mathrm{T}}\left(\bar{C}_{\mathrm{E}}, \Theta\right)+\right. \\
+\sqrt{\left.\left[X_{\mathrm{T}}\left(\bar{C}_{\mathrm{E}}, \Theta\right)-\bar{C}_{\mathrm{S}}\right]^{2}+4 \bar{K}_{\mathrm{M}} \bar{C}_{\mathrm{S}}\right]} \\
X_{\mathrm{T}}\left(\bar{C}_{\mathrm{E}}, \Theta\right)=\bar{K}_{\mathrm{M}}+\frac{k_{\mathrm{R}}^{\prime}}{k_{\mathrm{P}}} \bar{C}_{\mathrm{E}}
\end{gathered}
$$

It should be pointed out that the analyzed deactivation mechanism can be related to many enzymes currently applied in industrial practice $[17,18]$.

\section{SOLUTION OF THE MODEL}

When solving the above formulated model a process carried out at the optimal temperature control, guaranteeing maximum conversion $\alpha_{\mathrm{f}}=1-\bar{C}_{\mathrm{Sf}}$ or minimum time $t_{\mathrm{f}, \mathrm{opt}}$ indispensable for its achieving, has been considered. From the mathematical point of view both these variants of the optimal solutions are equivalent [19].

In industrial practice typical situation is encountered when a desired process is conducted starting from the initial substrate concentration $\bar{C}_{\mathrm{S}}\left(t=\underline{t_{0}}\right)=\bar{C}_{\mathrm{S} 0}$ and the initial biocatalyst activity $\bar{C}_{\mathrm{E}}\left(t=t_{0}\right)=\bar{C}_{\mathrm{E} 0}$ and terminating at the fixed final values of these variables $\bar{C}_{\mathrm{Sf}}, \bar{C}_{\mathrm{Ef}}$.

Optimal conditions provide the most advantageous solution for process control in view of the analyzed objective function. They have been determined based on the accomplishment of the optimization problem, which was aimed at finding a temperature as a function of time $T_{\text {opt }}(t)=1 / \Theta(t)$ so that for the initial conditions of the substrate concentration $\bar{C}_{\mathrm{S}}\left(t=t_{0}\right)=\bar{C}_{\mathrm{S} 0}$ and the enzyme activity $\bar{C}_{\mathrm{E}}\left(t=t_{0}\right)=\bar{C}_{\mathrm{E} 0}-$ in particular $\bar{C}_{\mathrm{E} 0}=1, \bar{C}_{\mathrm{S} 0}=1$ - as well as the appropriate fixed final values of the state variables $\bar{C}_{\mathrm{Sf}}$ and $\bar{C}_{\mathrm{Ef}}$ will minimize the process duration time $t_{\text {f,opt }}$.

Hence the initial and the final conditions for the state variables are defined as follows:

$$
\begin{gathered}
\bar{C}_{\mathrm{S}}(t=0)=\bar{C}_{\mathrm{S} 0}, \bar{C}_{\mathrm{S}}\left(t=t_{\mathrm{f}}\right)=\bar{C}_{\mathrm{Sf}} \\
\bar{C}_{\mathrm{E}}(t=0)=\bar{C}_{\mathrm{E} 0}, \bar{C}_{\mathrm{E}}\left(t=t_{\mathrm{f}}\right)=\bar{C}_{\mathrm{Ef}}
\end{gathered}
$$

In solving the formulated problem a classical method of variational calculus [20] has been applied to leading directly to differential equation describing a stationary temperature profile (Appendix A). For the analyzed problem the following equation is obtained

$$
\begin{gathered}
\frac{\mathrm{d} T}{\mathrm{~d} t}=-T^{2}\left[\frac{\partial f}{\partial \bar{C}_{\mathrm{E}}}+Z\left(\frac{\partial f}{\partial \bar{C}_{\mathrm{S}}}-\frac{\partial g}{\partial \bar{C}_{\mathrm{E}}}\right)+\right. \\
\left.-Z^{2} \frac{\partial g}{\partial \bar{C}_{\mathrm{S}}}-g \frac{\partial Z}{\partial \bar{C}_{\mathrm{E}}}-f \frac{\partial Z}{\partial \bar{C}_{\mathrm{S}}}-\frac{\partial Z}{\partial t}\right] \cdot\left(\frac{\partial Z}{\partial \Theta}\right)^{-1}
\end{gathered}
$$

$Z$ denotes Horn's variable defined as

$$
Z\left(t, \bar{C}_{\mathrm{S}}, \bar{C}_{\mathrm{E}}, \Theta\right)=\frac{\partial f}{\partial \Theta} / \frac{\partial g}{\partial \Theta}
$$

which according to the analyzed model takes the form

$$
\begin{gathered}
Z\left(t, \bar{C}_{\mathrm{S}}, \bar{C}_{\mathrm{E}}, \Theta\right)=u(\Theta) \cdot v\left[\bar{C}_{\mathrm{SIn}}\left(\bar{C}_{\mathrm{S}}, \bar{C}_{\mathrm{E}}, \Theta\right), \Theta\right] \\
\cdot w\left[\bar{C}_{\mathrm{SIn}}\left(\bar{C}_{\mathrm{S}}, \bar{C}_{\mathrm{E}}, \Theta\right), \Theta\right]
\end{gathered}
$$

where

$$
\begin{gathered}
u(\Theta)=\frac{k_{\mathrm{R}}^{\prime}}{k_{\mathrm{D}}} \\
v\left[\bar{C}_{\mathrm{SIn}}\left(\bar{C}_{\mathrm{S}}, \bar{C}_{\mathrm{E}}, \Theta\right), \Theta\right]= \\
=\left[\frac{\bar{K}_{\mathrm{D}}+\bar{C}_{\mathrm{SIn}}\left(C_{\mathrm{E}}, \bar{C}_{\mathrm{S}}, \Theta\right)}{\bar{K}_{\mathrm{M}}+\bar{C}_{\mathrm{SIn}}\left(C_{\mathrm{E}}, \bar{C}_{\mathrm{S}}, \Theta\right)}\right]^{2}
\end{gathered}
$$




$$
\begin{gathered}
w\left[\bar{C}_{\operatorname{SIn}}\left(C_{\mathrm{S}}, C_{\mathrm{E}}, \Theta\right), \Theta\right]= \\
=\frac{\left[E_{\mathrm{R}} \bar{C}_{\operatorname{SIn}}\left(\bar{K}_{\mathrm{M}}+\bar{C}_{\mathrm{SIn}}\right)-R \bar{K}_{\mathrm{M}} \frac{\partial \bar{C}_{\mathrm{SIn}}}{\partial \Theta}\right]}{\left[E_{\mathrm{D}} \bar{C}_{\operatorname{SIn}}\left(\bar{K}_{\mathrm{M}}+\bar{C}_{\mathrm{SIn}}\right)-R \bar{K}_{\mathrm{D}} \frac{\partial \bar{C}_{\mathrm{SIn}}}{\partial \Theta}\right]}
\end{gathered}
$$

The derivatives of Horn's variable $Z$ with respect to control variable $\Theta$, biocatalyst activity $\bar{C}_{\mathrm{E}}$ and substrate concentration $\bar{C}_{\mathrm{S}}$ are expressed

$$
\frac{\partial Z}{\partial y}=v w \frac{\partial u}{\partial y}+u w \frac{\partial v}{\partial y}+u v \frac{\partial w}{\partial y} \quad\left(y=\Theta, \bar{C}_{\mathrm{E}}, \bar{C}_{\mathrm{S}}\right)
$$

where

$$
\begin{gathered}
\frac{\partial u}{\partial \Theta}=\frac{\left(E_{\mathrm{D}}-E_{\mathrm{R}}\right)}{R} \cdot u \\
\frac{\partial v}{\partial y}=2 v \cdot \frac{\left(\bar{K}_{\mathrm{M}}-\bar{K}_{\mathrm{D}}\right)}{\left(\bar{K}_{\mathrm{D}}+\bar{C}_{\mathrm{SIn}}\right)\left(\bar{K}_{\mathrm{M}}+\bar{C}_{\mathrm{SIn}}\right)} \frac{\partial \bar{C}_{\mathrm{SIn}}}{\partial y} \\
\frac{\partial w}{\partial y}=R \bar{C}_{\operatorname{SIn}} \frac{\left[E_{\mathrm{R}} \bar{K}_{\mathrm{D}}\left(\bar{K}_{\mathrm{M}}+\bar{C}_{\mathrm{SIn}}\right)-E_{\mathrm{D}} \bar{K}_{\mathrm{M}}\left(\bar{K}_{\mathrm{D}}+\bar{C}_{\mathrm{SIn}}\right)\right]}{\left[E_{\mathrm{D}} \bar{C}_{\mathrm{SIn}}\left(\bar{K}_{\mathrm{D}}+\bar{C}_{\mathrm{SIn}}\right)-R \bar{K}_{\mathrm{D}} \frac{\partial \bar{C}_{\mathrm{SIn}}}{\partial \Theta}\right]^{2}} \\
\frac{\partial^{2} \bar{C}_{\mathrm{SIn}}}{\partial \Theta \partial y}+\frac{\left[E_{\mathrm{R}}\left(\bar{K}_{\mathrm{M}}+2 \bar{C}_{\mathrm{SIn}}\right)-w \cdot E_{\mathrm{D}}\left(\bar{K}_{\mathrm{D}}+2 \bar{C}_{\mathrm{SIn}}\right)\right]}{\left[E_{\mathrm{D}} \bar{C}_{\operatorname{SIn}}\left(\bar{K}_{\mathrm{D}}+\bar{C}_{\mathrm{SIn}}\right)-R \bar{K}_{\mathrm{D}} \frac{\partial \bar{C}_{\mathrm{SIn}}}{\partial \Theta}\right]} \frac{\partial \bar{C}_{\mathrm{SIn}}}{\partial y}
\end{gathered}
$$

The remaining elements revealed in the Eq. (9) are described by the following expressions:

$$
\begin{gathered}
\frac{\partial f}{\partial \bar{C}_{\mathrm{E}}}=-\frac{k_{\mathrm{R}}^{\prime}}{\left(\bar{K}_{\mathrm{M}}+\bar{C}_{\mathrm{SIn}}\right)}\left[\bar{C}_{\mathrm{SIn}}+\frac{\bar{K}_{\mathrm{M}} \bar{C}_{\mathrm{E}}}{\left(\bar{K}_{\mathrm{M}}+\bar{C}_{\mathrm{SIn}}\right)} \frac{\partial \bar{C}_{\mathrm{SIn}}}{\partial \bar{C}_{\mathrm{E}}}\right] \\
\frac{\partial f}{\partial \bar{C}_{\mathrm{E}}}=-\frac{k_{\mathrm{D}}}{\left(\bar{K}_{\mathrm{D}}+\bar{C}_{\mathrm{SIn}}\right)}\left[\bar{C}_{\mathrm{SIn}}+\frac{\bar{K}_{\mathrm{D}} \bar{C}_{\mathrm{E}}}{\left(\bar{K}_{\mathrm{D}}+\bar{C}_{\mathrm{SIn}}\right)} \frac{\partial \bar{C}_{\mathrm{SIn}}}{\partial \bar{C}_{\mathrm{E}}}\right] \\
\frac{\partial g}{\partial \bar{C}_{\mathrm{S}}}=-k_{\mathrm{D}}^{\prime} \bar{K}_{\mathrm{D}} \frac{\bar{C}_{\mathrm{E}}}{\left(\bar{K}_{\mathrm{M}}+\bar{C}_{\mathrm{SIn}}\right)^{2}} \frac{\partial \bar{C}_{\mathrm{SIn}}}{\partial \bar{C}_{\mathrm{S}}} \\
\left(\bar{K}_{\mathrm{D}}+\bar{C}_{\mathrm{SIn}}\right)^{2} \frac{\partial \bar{C}_{\mathrm{SIn}}}{\partial \bar{C}_{\mathrm{S}}}
\end{gathered}
$$

where $f, g$ denote the right-hand sides of Eqns. (5a) and (5b), respectively.

The first $\partial \bar{C}_{\text {SIn }} / \partial \Theta, \partial \bar{C}_{\text {SIn }} / \partial \bar{C}_{\mathrm{E}}, \partial \bar{C}_{\text {SIn }} / \partial \bar{C}_{\mathrm{S}}$ and the second derivatives $\partial^{2} \bar{C}_{\mathrm{SIn}} / \partial \Theta^{2}, \partial^{2} \bar{C}_{\mathrm{SIn}} / \partial \Theta \partial \bar{C}_{\mathrm{E}}, \partial^{2} \bar{C}_{\mathrm{SIn}} / \partial \Theta \partial \bar{C}_{\mathrm{S}}$ appearing in Eqns. (17-22) are given as follows:

$$
\begin{aligned}
& \frac{\partial \bar{C}_{\mathrm{SIn}}}{\partial \Theta}=\frac{1}{2} \frac{k_{\mathrm{R}}^{\prime}}{k_{\mathrm{P}}}\left(\frac{E_{\mathrm{P}}-E_{\mathrm{R}}}{R}\right) \bar{C}_{\mathrm{E}} . \\
& \cdot\left[\frac{X_{\mathrm{T}}-\bar{C}_{\mathrm{S}}}{\sqrt{\left(X_{\mathrm{T}}-\bar{C}_{\mathrm{S}}\right)^{2}+4 \bar{K}_{\mathrm{M}} \bar{C}_{\mathrm{S}}}}-1\right] \\
& \frac{\partial \bar{C}_{\mathrm{SIn}}}{\partial \bar{C}_{\mathrm{S}}}=\frac{1}{2}\left[\frac{X_{\mathrm{T}}+\bar{C}_{\mathrm{S}}}{\sqrt{\left(X_{\mathrm{T}}-\bar{C}_{\mathrm{S}}\right)^{2}+4 \bar{K}_{\mathrm{M}} \bar{C}_{\mathrm{S}}}}+1\right] \\
& \frac{\partial \bar{C}_{\mathrm{SIn}}}{\partial \bar{C}_{\mathrm{E}}}=\frac{1}{2} \frac{k_{\mathrm{R}}^{\prime}}{k_{\mathrm{P}}}\left[\frac{X_{\mathrm{T}}-\bar{C}_{\mathrm{S}}}{\sqrt{\left(X_{\mathrm{T}}-\bar{C}_{\mathrm{S}}\right)^{2}+4 \bar{K}_{\mathrm{M}} \bar{C}_{\mathrm{S}}}}-1\right] \\
& \frac{\partial^{2} \bar{C}_{\mathrm{SIn}}}{\partial \Theta^{2}}=\frac{1}{2} \frac{k_{\mathrm{R}}^{\prime}}{k_{\mathrm{P}}}\left(\frac{E_{\mathrm{P}}-E_{\mathrm{R}}}{R}\right)^{2} \bar{C}_{\mathrm{E}} . \\
& \cdot\left[\frac{k_{\mathrm{R}}^{\prime}}{k_{\mathrm{P}}} \frac{4 \bar{K}_{\mathrm{M}} \bar{C}_{\mathrm{S}} \bar{C}_{\mathrm{E}}}{\sqrt{\left[\left(X_{\mathrm{T}}-\bar{C}_{\mathrm{S}}\right)^{2}+4 \bar{K}_{\mathrm{M}} \bar{C}_{\mathrm{S}}\right]^{3}}}+\right. \\
& \left.+\frac{X_{\mathrm{T}}-\bar{C}_{\mathrm{S}}}{\sqrt{\left(X_{\mathrm{T}}-\bar{C}_{\mathrm{S}}\right)^{2}+4 \bar{K}_{\mathrm{M}} \bar{C}_{\mathrm{S}}}}-1\right] \\
& \frac{\partial^{2} \bar{C}_{\mathrm{SIn}}}{\partial \Theta \partial \bar{C}_{\mathrm{E}}}=\frac{1}{2} \frac{k_{\mathrm{R}}^{\prime}}{k_{\mathrm{P}}}\left(\frac{E_{\mathrm{P}}-E_{\mathrm{R}}}{R}\right) . \\
& \cdot\left[\frac{k_{\mathrm{R}}^{\prime}}{k_{\mathrm{P}}} \frac{4 \bar{K}_{\mathrm{M}} \bar{C}_{\mathrm{S}} \bar{C}_{\mathrm{E}}}{\sqrt{\left[\left(X_{\mathrm{T}}-\bar{C}_{\mathrm{S}}\right)^{2}+4 \bar{K}_{\mathrm{M}} \bar{C}_{\mathrm{S}}\right]^{3}}}+\right. \\
& \left.+\frac{X_{\mathrm{T}}-\bar{C}_{\mathrm{S}}}{\sqrt{\left(X_{\mathrm{T}}-\bar{C}_{\mathrm{S}}\right)^{2}+4 \bar{K}_{\mathrm{M}} \bar{C}_{\mathrm{S}}}}-1\right] \\
& \frac{\partial^{2} \bar{C}_{\mathrm{SIn}}}{\partial \Theta \partial \bar{C}_{\mathrm{S}}}=-\bar{K}_{\mathrm{M}} \frac{k_{\mathrm{R}}^{\prime}}{k_{\mathrm{P}}}\left(\frac{E_{\mathrm{P}}-E_{\mathrm{R}}}{R}\right) \bar{C}_{\mathrm{E}} . \\
& \cdot \frac{\left(X_{\mathrm{T}}+\bar{C}_{\mathrm{S}}\right)}{\sqrt{\left[\left(X_{\mathrm{T}}-\bar{C}_{\mathrm{S}}\right)^{2}+4 \bar{K}_{\mathrm{M}} \bar{C}_{\mathrm{S}}\right]^{3}}}
\end{aligned}
$$

where $X_{\mathrm{T}}$ is described by Eq. (7).

The above given Expressions (9-28), together with the state Eq. (5) as well as the initial and the final conditions for the state variables (Eqns. (8)), compose the model of the process carried out under a stationary profile of the optimal temperature. The effect of the formulated optimization problem can be classified as a two-point boundary value problem which can only be solved using numerical methods.

The more accurate analysis has revealed that a decisive effect on the structure of the optimal temperature profile is exerted by the activation energy quotient $E$ and a relation 
between the levels of the allowable temperatures $T_{\min }$ and $T_{\text {max }}$, the initial $T_{0}$ and final $T_{\mathrm{f}, \text { stat }}$ temperature of the stationary temperature profile as well as the value of the isothermal process temperature $T_{\text {isot }}$ resulting from its limiting values (Eq. (8)).

If $T_{\min } \leq T_{0}$ and $T_{\max } \geq T_{\mathrm{f} \text {,stat }}$, then the optimal temperature profile is entirely a stationary one. If one of these conditions is not fulfilled, it may activate a corresponding constraint.

If the permissible temperatures obey the relations

$$
\begin{gathered}
T_{0}<T_{\min }<T_{\text {isot }} \\
T_{\text {isot }}<T_{\max }<T_{\text {f,stat }}
\end{gathered}
$$

then in an optimal process the following elements will subsequently appear: an isothermal part at a level $T=T_{\min }$. a stationary part $T_{\text {stat }}(t)$ and an isothermal part at a level $T=T_{\max }$ (the most frequent case).

If finally $T_{\max } \leq T_{\text {isot }}$ or $T_{\min } \geq T_{\text {isot }}$, the optimal policy is strictly an isothermal one.

\section{ANALYSIS OF RESULTS}

The most important part of the present study is to define the extreme temperature conditions that would minimize the total duration time of the biotransformation process, in particular decomposition of hydrogen peroxide by catalase in the yeast cells of Saccharomyces cerevisae, with parallel deactivation of biocatalyst. For this purpose the data collected during a laboratory study for the process under consideration carried out at a temperature $T=30^{\circ} \mathrm{C}$ and listed in Table 1 [21] have been used.

Table 1. Parameters used in calculation

\begin{tabular}{c|l}
\hline Parameter & Value \\
\hline$C_{\mathrm{S} 0}$ & $0.01 \mathrm{kmol} / \mathrm{m}^{3}$ \\
\hline$E_{\mathrm{R}}$ & $4900 \mathrm{~J} / \mathrm{mol}$ \\
\hline$E_{\mathrm{D}}$ & $61700 \mathrm{~J} / \mathrm{mol}$ \\
\hline$E_{\mathrm{P}}$ & $74300 \mathrm{~J} / \mathrm{mol}$ \\
\hline$k_{\mathrm{R}} C_{\mathrm{E} 0}$ & $0.3 \cdot 10^{-4} \mathrm{kmol} /\left(\mathrm{m}^{6} \cdot \mathrm{s}\right)$ \\
\hline$k_{\mathrm{D}}$ & $1.92 \cdot 10^{-4} 1 / \mathrm{s}$ \\
\hline$K_{\mathrm{D}}$ & $0.016 \mathrm{kmol} / \mathrm{m}^{3}$ \\
\hline$K_{\mathrm{M}}$ & $0.083 \mathrm{kmol} / \mathrm{m}^{3}$ \\
\hline
\end{tabular}

For a value of $E_{\mathrm{R}}$ the computations have been expanded by adding the values of the activation energy of the deactivation process $E_{\mathrm{D}}$ for which the quotient
$E=E_{\mathrm{D}} / E_{\mathrm{R}}$ is within the limits from 1.5 to about 13 . The values of this quotient $E$ refer to the majority of the processes that are encountered in industrial practice [22], thus providing a generalization for the range of the present considerations.

The coefficients of frequency $k_{\mathrm{D} 0}$, corresponding to the assumed values of $E_{\mathrm{D}}$ in calculations, have been obtained based on the half-time decrease of biocatalyst activity, determined from the kinetic data and accounted in the solution of the mathematical model (5).

As it followed from the earlier investigations [23], high values of the quotient of the activation energy $E=E_{\mathrm{D}} / E_{\mathrm{R}}$ resulting from the kinetic data presented in Table 1 and the low values of the final activities $\bar{C}_{\text {Ef }}$ caused a rapid temperature increase at the final stage of the process and this justifies a need to apply the upper temperature limit. Thus, in the computations, a typical range of temperature applied for the majority of biological catalysts, i.e. with the lower and the upper temperatures $T_{\min }=293 \mathrm{~K}$ and $T_{\max }=323 \mathrm{~K}$, respectively, has been assumed.

The optimal temperature profiles $T_{\mathrm{opt}}(\tau)$ together with the corresponding variations of biocatalyst activity $\bar{C}_{\text {E,opt }}(\tau)$ for the selected values of the quotient $E$ are shown in Figs. 1-3. Because of a small effect of a parameter $E$ on the changes of the substrate concentration with time, the optimal concentration profile $\bar{C}_{\mathrm{S} \text {,opt }}(\tau)$ has additionally been inserted in Fig. 3 only for a selected value of $E$ and $E_{\mathrm{P}}$.

In contrast to processes with biocatalyst deactivation independent of substrate concentration the optimal temperature profiles for processes with parallel deactivation usually contain at least the upper temperature constrain

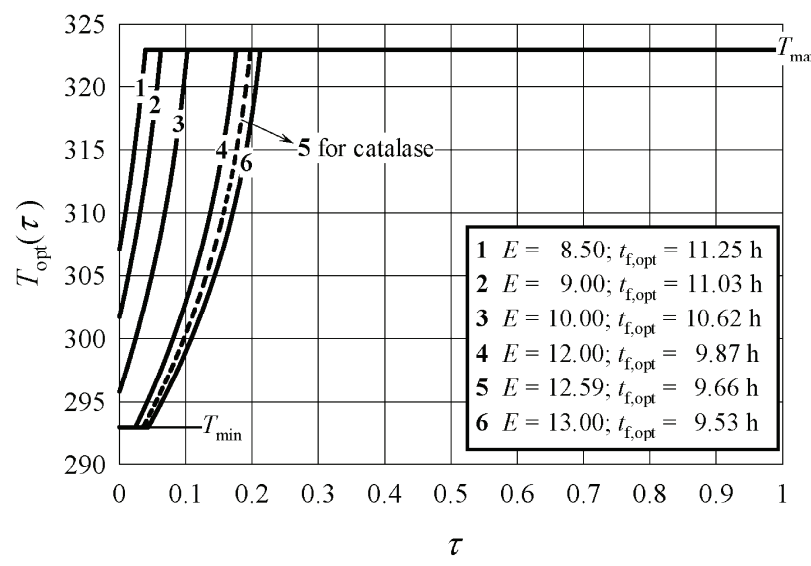

Fig. 1. Effect of the quotient of the activation energy $E$ on the optimal temperature profiles $T_{\text {opt }}(\tau)$ for the effectiveness factor $\eta=0.5$, the activation energy for permeability of the cells membrane $E_{\mathrm{P}}=E_{\mathrm{R}}$ as well as the final values of biocatalyst activity and substrate concentration equal to $\bar{C}_{\mathrm{Ef}}=0.1$ and $\bar{C}_{\text {Sf }}=0.1$, respectively 


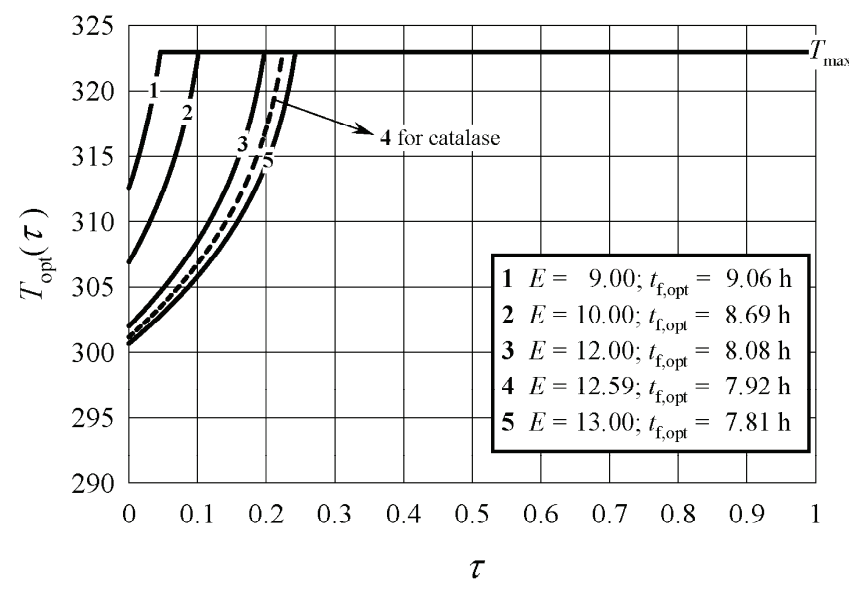

Fig. 2. Effect of the quotient of the activation energy $E$ on the optimal temperature profiles $T_{\text {opt }}(\tau)$ for the effectiveness factor $\eta=0.5$, the activation energy for permeability of the cells membrane $E_{\mathrm{P}}=74.3 \mathrm{~kJ} / \mathrm{mol}$ as well as the final values of biocatalyst activity and substrate concentration equal to $\bar{C}_{\mathrm{Ef}}=0.1$ and $\bar{C}_{\mathrm{Sf}}=0.1$, respectively

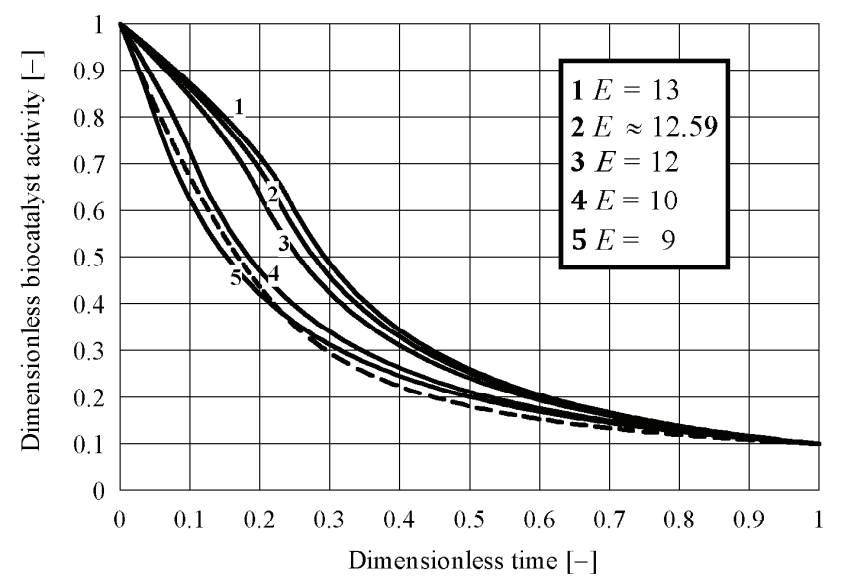

Fig. 3. Effect of the quotient of the activation energy $E$ on the variations of biocatalyst activity in the optimal conditions $\bar{C}_{\mathrm{E}, \text { opt }}(\tau)$ for the effectiveness factor $\eta=0.5$, the activation energy for permeability of the cells membrane $E_{\mathrm{P}}=74.3 \mathrm{~kJ} / \mathrm{mol}$. A dashed line describes substrate concentration variations $\bar{C}_{\mathrm{S} \text {,opt }}(\tau)$ for $E=12.59 \mathrm{~kJ} / \mathrm{mol}, E_{\mathrm{P}}=74.3 \mathrm{~kJ} / \mathrm{mol}$ and the final biocatalyst activity $\bar{C}_{\mathrm{Ef}}=0.1$

$T=T_{\max }$. It should be pointed out, however, that at low values of the parameter $E$ the profiles of optimal temperature assume the range of its greater values. In effect, at such values of $E$ and at the active temperature constraints, the optimal temperature policy is usually strictly isothermal at the level $T=T_{\max }$.

The presence of the cell membrane is represented by the effectiveness factor $\eta$ [24-27] of enzyme contained within the cells. This effectiveness factor is defined as ratio of the reaction rate at substrate concentration inside a cell (observed reaction rate) to the reaction rate at substrate concentration in a fluid core.

An impact of the effectiveness factor $\eta$ on the profile of optimal temperature is depicted in Figs. 4 and 5. The frequency coefficient $k_{\mathrm{P} 0}$ encountered in this study can be related to the effectiveness factor $\eta$ of the enzyme contained within the cells and is given by the following equation:

$k_{\mathrm{P} 0}=\frac{\eta}{(1-\eta)} \frac{k_{\mathrm{R} 0} C_{\mathrm{E} 0}}{C_{\mathrm{S} 0}} \frac{\left[\bar{K}_{\mathrm{M}}+(1-\eta) \bar{C}_{\mathrm{S}}\right]}{\left(\bar{K}_{\mathrm{M}}+\bar{C}_{\mathrm{S}}\right)^{2}} \bar{C}_{\mathrm{E}} \exp \left(\frac{E_{\mathrm{P}}-E_{\mathrm{R}}}{R T^{*}}\right)$

in which $T^{*}$ denotes standard temperature.

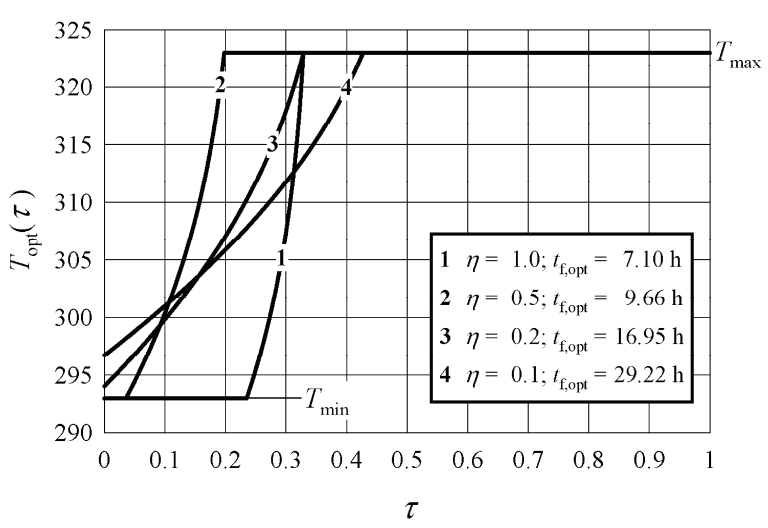

Fig. 4. Effect of the effectiveness factor $\eta$ on the optimal temperature profiles $T_{\text {opt }}(\tau)$ for the quotient of the activation energy $E=12.59$ (Table 1), the activation energy for permeability of the cells membrane $E_{\mathrm{P}}=E_{\mathrm{R}}$ as well as the final values of biocatalyst activity and substrate concentration equal to $\bar{C}_{\mathrm{Ef}}=0.1$ and $\bar{C}_{\mathrm{Sf}}=0.1$, respectively

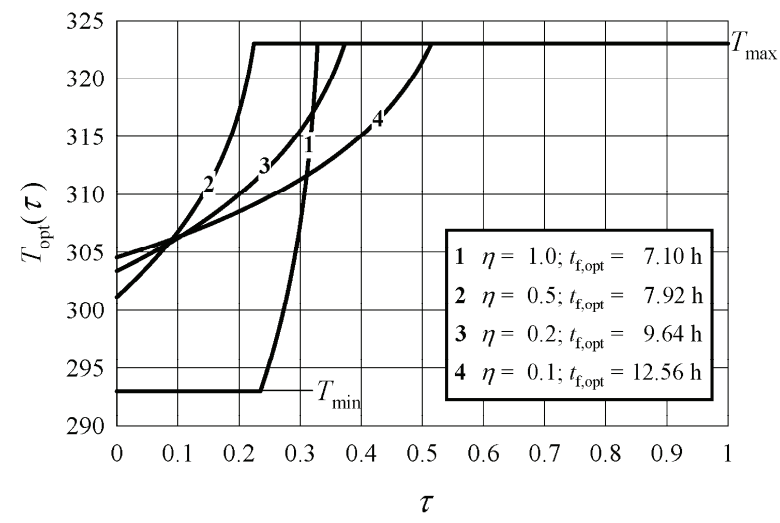

Fig. 5. Effect of the effectiveness factor $\eta$ on the optimal temperature profiles $T_{\text {opt }}(\tau)$ for the quotient of the activation energy $E=12.59$ (Table 1), the activation energy for permeability of the cells membrane $E_{\mathrm{P}}=74.3 \mathrm{~kJ} / \mathrm{mol}$ as well as the final values of biocatalyst activity and substrate concentration equal to $\bar{C}_{\mathrm{Ef}}=0.1$ and $\bar{C}_{\mathrm{Sf}}=0.1$, respectively 
A value of $\eta \rightarrow 1$ corresponds to a case where the diffusional resistance does not affect the reaction rate. In the case of low concentration, for which $C_{\mathrm{S}}<<K_{\mathrm{M}}$, Eq. 29 simplifies to that used in the work [23].

Since temperature significantly affects transfer processes across the cell wall, the courses of the optimal temperature profiles $T_{\mathrm{opt}}(\tau)$ at different values of the activation energy $E_{\mathrm{P}}$ of the transfer process across the cell wall are displayed in Figs. 6 and 7.

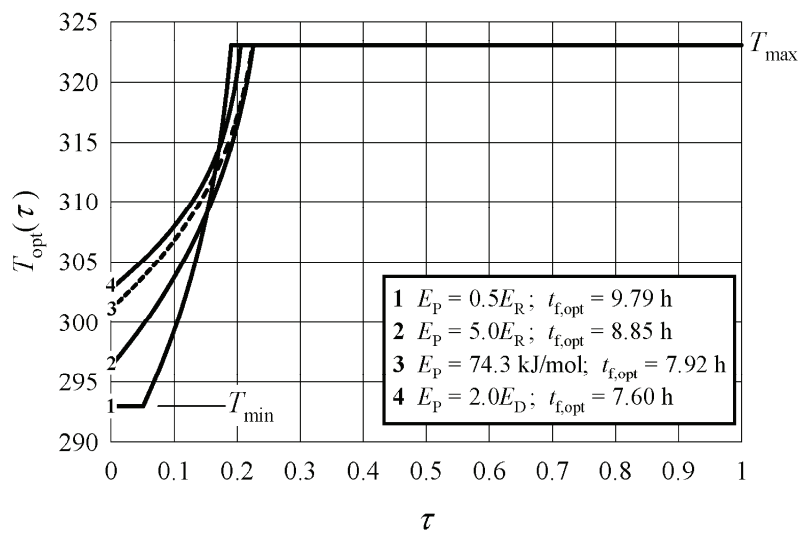

Fig. 6. Effect of the activation energy for permeability of the cells membrane $E_{\mathrm{P}}$ on the optimal temperature profiles $T_{\mathrm{opt}}(\tau)$ for the quotient of the activation energy $E=12.59$, a value of the effectiveness factor $\eta=0.5$ as well as the final values of biocatalyst activity and substrate concentration equal to $\bar{C}_{\mathrm{Ef}}=0.1$ and $\bar{C}_{\mathrm{Sf}}=0.1$, respectively

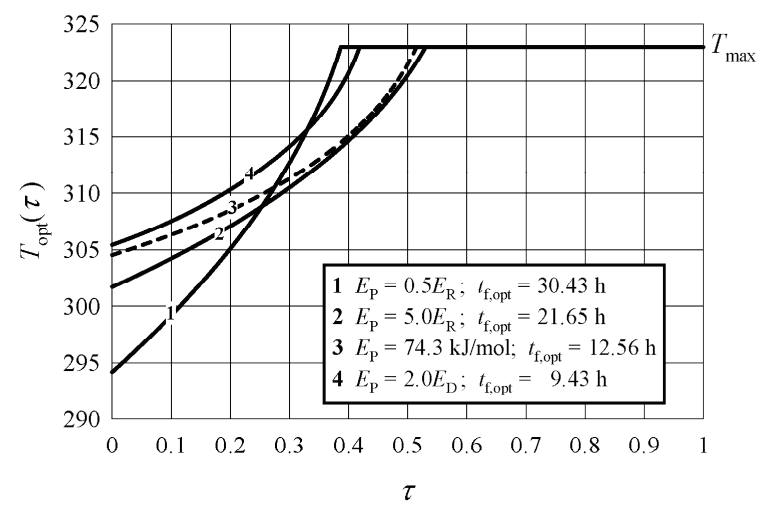

Fig. 7. Effect of the activation energy for permeability of the cells membrane $E_{\mathrm{P}}$ on the optimal temperature profiles $T_{\mathrm{opt}}(\tau)$ for the quotient of the activation energy $E=12.59$, a value of the effectiveness factor $\eta=0.1$ as well as the final values of biocatalyst activity and substrate concentration equal to $\bar{C}_{\text {Ef }}=0.1$ and $\bar{C}_{\mathrm{Sf}}=0.1$, respectively

As it follows from Figs. 6 and 7, the variations of temperature with time $T_{\text {opt }}(\tau)$ are located at higher values for increasing values of $E_{\mathrm{p}}$. In consequence, the lower constraint becomes inactive and the total process time $t_{\mathrm{f}, \mathrm{opt}}$ decreases.

Optimal temperature control accompanying biotransformation processes frequently hold in their structure at least an upper temperature constraint. However, it is worth mentioning that in case of processes with deactivating biocatalyst independent of the substrate concentration the optimal temperature profiles may not be constrained by permissible temperatures $T=T_{\min }, T=T_{\max }$ and usually are monotonically increasing the time function. In particular, this becomes evident for $E_{\mathrm{D}}>E_{\mathrm{P}}$. However, at $E_{\mathrm{D}} \leq E_{\mathrm{P}}$ and low values of biocatalyst effectiveness $(\eta \leq 0.2)$ a course of a stationary optimal temperature atypical for enzymatic processes is seen (Grubecki, 1997).

Actually, at slightly higher final activities, e.g. $\bar{C}_{\text {Ef }}=0.3$ one can notice an appearance of temperature minimum $(\eta=0.2)$ or its decrease $(\eta=0.1)$ taking place for the entire process duration time.

A decrease in the final biocatalyst activity $\left(\bar{C}_{\mathrm{Ef}}=0.1\right)$ results in the course of the temperature profiles within the range of higher values, giving rise to a situation that a temperature minimum becomes visible. It is moving towards the end of the process as $\eta$ decreases.

\section{CONCLUSIONS}

An analysis aiming to search for an optimal temperature control ensuring the shortest duration time of a biotransformation process with a parallel deactivation of enzyme encapsulated inside the microorganisms cells has been presented.

The conditions required for conducting a biotransformation at the optimal temperature were established. It was found that, for a process occurring with a biocatalyst deactivation both parallel and independent of the substrate concentration, the temperature profile was increasing with time. For the parallel deactivation it was necessary to account for, at least, the upper temperature constraint $T=T_{\max }$.

Application of microorganisms cells results in slowing down the reaction rate and shifting the initial temperature of the stationary profile to higher values. They are more pronounced the lower permeability of the cell membrane $(\eta)$ is. In consequence, an extension of the process duration time is observed along the sections of the optimal profile while the lower temperature constraint usually becomes inactive.

Furthermore, lowering values of the parameter $E$ causes the optimal temperature profiles shift towards higher values. In effect, optimal control consists of the following 
subsequent parts: isothermal $T=T_{\min }$, stationary $T_{\text {stat }}(t)$ and isothermal $T=T_{\max }$, at high values of $E$, throughout the profiles with an inactive lower temperature constraint or becomes strictly isothermal policy at relatively low values of $E$.

\section{References}

[1] N. Aziz, I.M. Mujtaba, Optimal operation policies in batch reactors. Chemical Engineering Journal 85, 313-325 (2002).

[2] N.M. Faqir, Optimization of operating temperature for an continuous immobilized glucose isomerase reactor with pseudo linear kinetics. Engineering in Life Sciences 4, 450459 (2004).

[3] A. Arpornwichanop, P. Kittisupakorn, I.M. Mujtaba, Online dynamic optimization and control strategy for improving the performance of batch reactors. Chemical Engineering and Processing 44, 101-114 (2005).

[4] A. Sadana, Generalized optimum temperature operations criterion for a deactivating immobilized enzyme batch reactor. AIChE Journal 25, 535-538 (1979).

[5] Z. Szwast, S. Sieniutycz, Optimal temperature profiles for parallel-consecutive reactions with deactivating catalyst. Catalysis Today 66, 461-466 (2001).

[6] E. Cepeda, M. Hermosa, A. Ballesteros, Optimization of maltodextrin hydrolysis by glucoamylase in a batch reactor. Biotechnology and Bioengineering 76, $70-76$ (2001).

[7] Z. Szwast, S. Sieniutycz, Optimal dynamical processes in tubular reactor with deactivation of multi-run moving catalyst, Chemical Engineering Journal 103, 45-50 (2004).

[8] N.M. Faqir, I.M. Abu-Reesh, Optimum temperature operation mode for glucose isomerase reactor operating at constant glucose conversion. Bioprocess Engineering 19, 11-17 (1998).

[9] N.M. Faqir, M.M. Attarakin, Optimal temperature policy for immobilized enzyme packed bed reactor performing reversible Michaelis-Menten kinetics using the disjoint policy. Biotechnology and Bioengineering 77, 163-173 (2002).

[10] J.L. Gómez, A. Bódalo, E. Gómez, J. Bastida, M.F. Maximo, Two-parameter model for effectiveness factor for immobilized enzymes with reversible Michaelis-Menten kinetics. Chemical Engineering Science 58, 4287-4290 (2003).

[11] R. Khalilpour, R. Roostaazad, Development and verification of a model to describe an immobilized glucose isomerase packed bed bioreactor, Biochemical Engineering Journal 40, 328-336 (2008).

[12] A.E. Al-Muftah, I. M. Abu-Reesh, Effects of internal mass transfer and product inhibition on a simulated immobilized enzyme-catalyzed reactor for lactose hydrolysis. Biochemical Engineering Journal 23, 139-153 (2005).
[13] E.J. Mammarella, A.C. Rubiolo, Effect of biocatalyst swelling on the operation of packed-bed immobilized enzyme bioreactor. Process Biochemistry 44, 183-190 (2009).

[14] S. Mudliar, S. Banerjee, A. Vaidya, S. Devotta, Steady state model for evaluating of external and internal mass transfer effects in an immobilized biofilm. Bioresource Technology 99, 3468-3474 (2008).

[15] A.E. Al-Muftah, I. M. Abu-Reesh, Effects of simultaneous internal and external mass transfer and product inhibition on immobilized enzyme-catalyzed reactor. Biochemical Engineering Journal 27, 167-178 (2005).

[16] D.D. Do, R.H. Weiland, Consistency between rate expressions for enzyme reactions and deactivation. Biotechnology and Bioengineering 22, 1087-1093 (1980).

[18] K. Nazari, A. Mahmoudi, M. Shahrooz, R. Khodafarin, A.A. Moosavi-Movahedi, Suicide-peroxide inactivation of horseradish peroxidase in the presence of Sodium nDodecyl Sulophate: A study of the enzyme deactivation kinetics. Journal of Enzyme Inhibition and Medicinal Chemistry 20, 285-292 (2005).

[18] S. Chazarra, J. Cabanes, J. Escribano, F. Garcia-Carmona, Kinetic study of the suicide inactivation of latent polyphenoloxidase from iceberg lettuce (Lactuca sativa) induced by 4-tert-butylcatechol in the presence of SDS. Biochimica et Biophysica Acta 1339, 297-303 (1997).

[19] S. Szepe, O. Levenspiel, Optimal temperature policies for reactors subject to catalyst deactivation - I batch reactor. Chemical Engineering Science 23, 881-894 (1968).

[20] E. Pinch, Optimal Control and the Calculus of Variations. Oxford: Oxford University Press 1998.

[21] M. Wójcik, Kinetics of catalase deactivation of yeast Saccharomyces cerevisiae by hydrogen peroxide. Bydgoszcz: Publishing of University of Technology and Agriculture 1988 (in Polish).

[22] S.H. Lin, Optimal Feed Temperature for an immobilized Enzyme Packed-Bed Reactor. J. Chem. Tech. Biotechnol. 50, 17-26 (1991).

[23] I. Grubecki, An Analysis of the temperature policy for enzymatic reaction with Michaelis-Menten kinetics in a batch reactor. Ph.D. Thesis, Faculty of Chemical and Process Engineering, University of Technology, Warsaw 1997 (in Polish).

[24] M. Al-Shanag, Z. Al-Qodah, J. Herrero, J.A.C. Humphrey, F. Giralt, Using a wall-driven flow to reduce the external mass-transfer resistance of a bioreaction system. Biochemical Engineering Journal 39, 554-565 (2008).

[25] M. Szukiewicz, R. Petrus, Approximate model for diffusion and reaction in a porous pellet and an effectiveness factor, Chemical Engineering Science 59, 479-483 (2004).

[26] M.L. Shuler, F. Kargi, Bioprocess Engineering: Basic Concepts. Prentice Hall, New Jersey 2002.

[27] K. van't Riet, J. Tramper, Basic Bioreactor Design. Marcel Dekker, Inc., New York, Basel, Hong Kong 1991. 


\section{APPENDIX A}

Derivation of the equation for stationary optimal decision using variational method

Let

$$
\begin{aligned}
& \frac{\mathrm{d} \bar{C}_{\mathrm{S}}}{\mathrm{d} t}=f\left(t, \bar{C}_{\mathrm{S}}, \bar{C}_{\mathrm{E}}, \Theta\right) \\
& \frac{\mathrm{d} \bar{C}_{\mathrm{E}}}{\mathrm{d} t}=g\left(t, \bar{C}_{\mathrm{S}}, \bar{C}_{\mathrm{E}}, \Theta\right)
\end{aligned}
$$

determines an equation of state in an optimization problem minimizing the process duration time. Now, the objective function takes the following form

$$
S=\int_{0}^{t_{\mathrm{f}}} \mathrm{d} t
$$

which is a special case of the Bolza functional and determines the so-called Lagrange problem.

Since the mathematical model (Eqs. (A.1) and (A.2)) is dependent on the two state variables $\left(\bar{C}_{\mathrm{E}}, \bar{C}_{\mathrm{S}}\right)$ and one decisive variable $(\Theta)$, then when solving the problem it is possible to determine a differential equation that describes a stationary control.

For this purpose, by introducing the Lagrange multipliers $\lambda_{i}(t) \quad(i=\mathrm{E}, \mathrm{S})$ it is indispensable to formulate a modified objective function $S_{\mathrm{R}}$ defined as

$$
\begin{aligned}
S_{\mathrm{R}} & =\int_{0}^{t_{\mathrm{f}}}\left\{1+\lambda_{\mathrm{S}}(t)\left[f\left(t, \bar{C}_{\mathrm{S}}, \bar{C}_{\mathrm{E}}, \Theta\right)-\frac{\mathrm{d} \bar{C}_{\mathrm{S}}}{\mathrm{d} t}\right]+\right. \\
& \left.+\lambda_{\mathrm{E}}(t)\left[g\left(t, \bar{C}_{\mathrm{S}}, \bar{C}_{\mathrm{E}}, \Theta\right)-\frac{\mathrm{d} \bar{C}_{\mathrm{E}}}{\mathrm{d} t}\right]\right\} \mathrm{d} t
\end{aligned}
$$

which will undergo the minimization procedure. The functions $\bar{C}_{\text {E,stat }}(t)$ and $\bar{C}_{\mathrm{S} \text {,stat }}(t)$ that minimize this functional are searched as the solutions of the Euler-Lagrange equations being the necessary condition for the existence of the functional (A.4) [20]

$$
\frac{\mathrm{d}}{\mathrm{d} t}\left[\frac{\partial L}{\partial(\mathrm{d} y / \mathrm{d} t)}\right]-\frac{\partial L}{\partial y}=0 \quad\left(y=\bar{C}_{\mathrm{E}}, \bar{C}_{\mathrm{S}}, \Theta\right)
$$

where $L\left(\bar{C}_{\mathrm{E}}, \bar{C}_{\mathrm{S}}, \Theta, \mathrm{d} \bar{C}_{\mathrm{E}} / \mathrm{d} t, \mathrm{~d} \bar{C}_{\mathrm{S}} / \mathrm{d} t\right)$ is a function expressed in form of

$$
\begin{aligned}
L & =1+\lambda_{\mathrm{S}}(t)\left[f\left(t, \bar{C}_{\mathrm{S}}, \bar{C}_{\mathrm{E}}, \Theta\right)-\frac{\mathrm{d} \bar{C}_{\mathrm{S}}}{\mathrm{d} t}\right]+ \\
& +\lambda_{\mathrm{E}}(t)\left[g\left(t, \bar{C}_{\mathrm{S}}, \bar{C}_{\mathrm{E}}, \Theta\right)-\frac{\mathrm{d} \bar{C}_{\mathrm{E}}}{\mathrm{d} t}\right]
\end{aligned}
$$

Calculating the variables that appear in Eq. (A.5) yields

$$
\begin{gathered}
\frac{\mathrm{d} \lambda_{\mathrm{S}}}{\mathrm{d} t}=-\lambda_{\mathrm{S}}(t) \frac{\partial f\left(t, \bar{C}_{\mathrm{E}}, \bar{C}_{\mathrm{S}}, \Theta\right)}{\partial \bar{C}_{\mathrm{S}}}+ \\
-\lambda_{\mathrm{E}}(t) \frac{\partial g\left(t, \bar{C}_{\mathrm{E}}, \bar{C}_{\mathrm{S}}, \Theta\right)}{\partial \bar{C}_{\mathrm{S}}} \\
\frac{\mathrm{d} \lambda_{\mathrm{E}}}{\mathrm{d} t}=-\lambda_{\mathrm{S}}(t) \frac{\partial f\left(t, \bar{C}_{\mathrm{E}}, \bar{C}_{\mathrm{S}}, \Theta\right)}{\partial \bar{C}_{\mathrm{E}}}+ \\
-\lambda_{\mathrm{E}}(t) \frac{\partial g\left(t, \bar{C}_{\mathrm{E}}, \bar{C}_{\mathrm{S}}, \Theta\right)}{\partial \bar{C}_{\mathrm{E}}} \\
-\lambda_{\mathrm{S}}(t) \frac{\partial f\left(t, \bar{C}_{\mathrm{E}}, \bar{C}_{\mathrm{S}}, \Theta\right)}{\partial \Theta}+ \\
-\lambda_{\mathrm{E}}(t) \frac{\partial g\left(t, \bar{C}_{\mathrm{E}}, \bar{C}_{\mathrm{S}}, \Theta\right)}{\partial \Theta}=0
\end{gathered}
$$

From the above set of Eqs. (A.7)-(A.9) it is possible to eliminate the multipliers $\lambda_{\mathrm{E}}(t)$ and $\lambda_{\mathrm{S}}(t)$. Assessing the multiplier $\lambda_{\mathrm{E}}(t)$ from Eq. (A.9)

$$
\lambda_{\mathrm{E}}(t)=-\lambda_{\mathrm{S}}(t) \frac{\partial f\left(t, \bar{C}_{\mathrm{E}}, \bar{C}_{\mathrm{S}}, \Theta\right)}{\partial g\left(t, \bar{C}_{\mathrm{E}}, \bar{C}_{\mathrm{S}}, \Theta\right)}
$$

and accounting for its value in Eq. (A.8), then dividing it side by side by $\lambda_{\mathrm{S}}(t)$ one can get (for clarity of the notation in some of the expressions of the further part of this derivation it is assumed

$$
\begin{gathered}
f\left(t, \bar{C}_{\mathrm{E}}, \bar{C}_{\mathrm{S}}, \Theta\right)=f, \quad g\left(t, \bar{C}_{\mathrm{E}}, \bar{C}_{\mathrm{S}}, \Theta\right)=g, \\
\left.Z\left(t, \bar{C}_{\mathrm{E}}, \bar{C}_{\mathrm{S}}, \Theta\right)=Z\right) \\
\frac{\mathrm{d} Z}{\mathrm{~d} t}=\frac{\partial f}{\partial \bar{C}_{\mathrm{E}}}+Z\left(\frac{\partial f}{\partial \bar{C}_{\mathrm{S}}}-\frac{\partial g}{\partial \bar{C}_{\mathrm{E}}}\right)-Z^{2} \frac{\partial g}{\partial \bar{C}_{\mathrm{S}}}
\end{gathered}
$$

with

$$
Z=\frac{\partial f}{\partial \Theta} / \frac{\partial g}{\partial \Theta}
$$

and is called as the Horn's variable [26].

Making use of a definition of the complete derivative $Z$, with respect to time, a searched equation describing changes of the stationary decision $\Theta$ is gained in time

$$
\begin{gathered}
\frac{\mathrm{d} \Theta}{\mathrm{d} t}=\left[\frac{\partial f}{\partial \bar{C}_{\mathrm{E}}}+Z\left(\frac{\partial f}{\partial \bar{C}_{\mathrm{S}}}-\frac{\partial g}{\partial \bar{C}_{\mathrm{E}}}\right)+\right. \\
\left.-Z^{2} \frac{\partial g}{\partial \bar{C}_{\mathrm{S}}}-g \frac{\partial Z}{\partial \bar{C}_{\mathrm{E}}}-f \frac{\partial Z}{\partial \bar{C}_{\mathrm{S}}}-\frac{\partial Z}{\partial t}\right] \cdot\left(\frac{\partial Z}{\partial \Theta}\right)^{-1}
\end{gathered}
$$




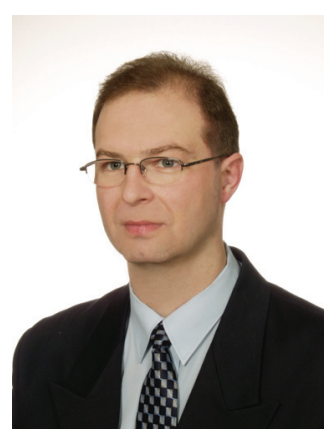

DR. IRENEUSZ GRUBECKI is a senior lecturer in the Department of Chemical and Bioprocess Engineering at Bydgoszcz University of Technology and Life Sciences. He graduated from the above university with an MSc degree in chemical engineering in 1991. In 1998 he received a $\mathrm{PhD}$ degree in chemical and process engineering from Warsaw University of Technology. The area of his specialization is modeling, design and optimization of biochemical reactors. He has published results of his research in national and international journals. 